\title{
Virtual Spatial Modulation for MIMO Systems
}

\author{
Xudong Zhu ${ }^{1}$, Zhaocheng Wang ${ }^{1}$, Qi Wang ${ }^{1}$, and Harald Haas ${ }^{2}$ \\ ${ }^{1}$ Tsinghua National Laboratory for Information Science and Technology (TNlist), Tsinghua University, China \\ ${ }^{2}$ University of Edinburgh, Institute for Digital Communications, United Kingdom \\ Email: zhuxd12@mails.tsinghua.edu.cn, zcwang@tsinghua.edu.cn, and h.haas@ed.ac.uk
}

\begin{abstract}
Compared with the conventional amplitude phase modulation (APM), spatial modulation (SM) is a low-complexity, yet energy-efficient transmission technique, whereby transmit antenna (TA) indices are utilized to convey the information. However, the number of the required TAs grows exponentially with the number of transmitted bits, which leads to unacceptable pilot overhead for channel estimation in practical systems. To reduce the number of TAs whereas keep the data rate unchanged, virtual spatial modulation (VSM) is proposed in the first time. Specifically, by activating multiple TAs with their corresponding analog phase shifters (APSs), massive equivalent channel vectors could be constructed based on the combinations of original channel vectors from different TAs and their phase rotations. By way of mapping each equivalent channel vector to a virtual transmit antenna (VTA) index which might convey the information, the number of the required TAs could grow linearly with the number of transmitted bits. Furthermore, the selection of a VTA subset from all available VTAs is formulated as a combinatorial optimization problem to maximize the minimal Euclidean distance (ED) among the equivalent channel vectors. A spatial constellation optimizing (SCO) algorithm is proposed to obtain a near-optimal solution to this problem with lowcomplexity. Simulation results demonstrate that the proposed VSM is able to achieve lower bit error rate (BER) under the same transmit rate compared with the conventional SM and APM schemes.
\end{abstract}

Key Words - Spatial modulation (SM), multiple-input multipleoutput system (MIMO), virtual spatial modulation (VSM).

\section{INTRODUCTION}

Multiple-input multiple-output (MIMO) technique in wireless communications is an effective way to improve capacity and reliability by exploiting multiplexing, diversity, and antenna gains [1], [2]. However, regardless of the use as spatial multiplexing, diversity or smart antennas system, the main drawback of any MIMO schemes is an increase in complexity and cost [5]. Specifically, the impact of inter-channel interference (ICI), the need for inter-antenna synchronization (IAS), and the requirement for multiple radio frequency (RF) chains are three common issues that lead to practical challenges for the introduction of MIMO in real-world systems [6]-[9].

To eliminate these practical limitations of MIMO systems, a new approach to exploit the spatial dimension has been introduced, which uses the transmit antenna (TA) indices to convey the information [5]-[9]. The main principle is often referred to as space modulation. Representative techniques are space shift keying (SSK) modulation where amplitude phase modulation (APM) is not required and only TA indices are utilized to convey the information [3], [5]. ICI and IAS are totally avoided in SSK which results in a significan$\mathrm{t}$ simplification in transceiver design and the reduction of decoding complexity [5]. Meanwhile, by way of combining APM with TA index modulation, a hybrid modulation scheme named spatial modulation (SM) is proposed, which creates a unique three dimensional constellation diagram (combining the conventional two-dimensional complex signal space with an orthogonal space dimension) [6]. To exploit high spectral efficiency (SE), several variations of generalized SM (GSM) have been proposed by activating several TAs simultaneously [7]-[9]. Various SM schemes provide their own sets of benefits and disadvantages [5]-[9], but generally suffer from two limitations for practical implementation as described in the following:

1) Channel Estimation: By adopting conventional pilot transmission for the estimation of channel state information (CSI) at the receiver, the pilot length of the above-mentioned SM schemes should be proportional to the number of TAs [5][9], and have to be transmitted from different TAs in a sequential way as there is only one RF chain [2]. Hence, there is an additional cost that comes with the reduction in hardware complexity in terms of additional channel estimation overhead for CSI. However, several methodologies have been proposed to overcome this limitation by exploiting antenna correlation [10].

2) Hardware Restriction: The transmit data rate of the conventional SM schemes is closely related to the number of TAs [5]-[9], e.g., the number of TAs has to be doubled to realize the increase of only $1 \mathrm{bps} / \mathrm{symbol}$. Hence, to support higher data rate, the number of TAs has to be increased exponentially, which leads to an economical hardware restriction in practical implementation, e.g., mobile handsets with limited space [5]-[9].

In this paper, we break away from the traditional SM schemes, which convey information on physical TA indices - instead, the concept of virtual spatial modulation (VSM) is proposed. By activating multiple TAs with analog phase shifters (APSs) [11], [12], massive virtual TAs (VTAs) could be constructed, whose equivalent channel vectors are the combinations of original channel vectors from physical TAs and their phase rotations. By way of conveying the information over VTA indices, the number of the required physical TAs could grow linearly with the number of transmitted bits. Furthermore, the selection of a VTA subset from all available VTAs is formulated as a combinatorial optimization problem to maximize the minimal Euclidean distance (ED) among the corresponding equivalent channel vectors. After that, a spatial constellation optimizing (SCO) algorithm is provided to obtain a near-optimal solution with low-complexity. Simulation 
results show that the proposed VSM scheme is able to achieve lower bit error rate (BER) for the same transmit rate compared with conventional SM and APM schemes.

\section{SYSTEM MODEL}

In this section, the general transceiver of the proposed VSM is introduced, whereby transmission, spatial constellation, and detection are addressed in detail.

\section{A. Transmission}

As illustrated in Fig. 1, the general system model of the proposed VSM consists of a MIMO wireless link with $N_{t}$ TAs and $N_{r}$ receive antennas (RAs). The constant signal $\frac{1}{\sqrt{N_{t}}}$ is up-converted by the single RF chain, and then uploaded to each APS. A random sequence of independent information bits $\mathbf{b}=\left[\begin{array}{llll}b_{1} & b_{2} & \cdots\end{array}\right]$ enters the VSM mapper with output signal to control the series of APSs, which are low-cost devices to realize quantized phase rotation for RF signals [11], [12]. By considering $B$ quantized APSs, the available phase rotation of such devices could be represented as

$$
\Theta=\left\{e^{j \frac{2 \pi n}{2^{B}}}: n=0,1, \cdots, 2^{B}-1\right\},|\Theta|=2^{B} .
$$

Hence, the modulation is carried out at the APS array, and the modulated signal vector could be represented as

$$
\mathbf{x}_{j}=\frac{1}{\sqrt{N_{t}}}\left[\begin{array}{llll}
\theta_{j, 1} & \theta_{j, 2} & \cdots & \theta_{j, N_{t}}
\end{array}\right]^{T}, 1 \leq j \leq 2^{B N_{t}},
$$

where $\theta_{j, k} \in \Theta$ denotes the $k$-th APS's phase rotation of the $j$-th combination branch in the proposed VSM. By adopting all available combinations of the APS array, i.e., $\left\{\mathbf{x}_{j}: 1 \leq\right.$ $\left.j \leq 2^{B N_{t}}\right\}$, the modulation order could be

$$
m_{\mathrm{VSM}}=\log _{2}\left(2^{B N_{t}}\right)=B N_{t},
$$

which is much larger than $m_{\mathrm{SSK}}=\log _{2}\left(N_{t}\right)$ of the conventional SSK scheme.

The modulated signal vector $\mathbf{x}_{j}$ with a power constraint of unity (i.e., $\mathrm{E}_{\mathbf{x}}\left\{\mathbf{x}_{j}^{H} \mathbf{x}_{j}\right\}=1$ ) is transmitted over a wireless channel $\mathbf{H}=\left[\begin{array}{llll}\mathbf{h}_{1} & \mathbf{h}_{2} & \cdots & \mathbf{h}_{N_{t}}\end{array}\right] \in \mathbb{C}^{N_{r} \times N_{t}}$, and experiences an $N_{r}$-dimensionality additive white Gaussian noise (AWGN) vector $\mathbf{n}=\left[\begin{array}{llll}n_{1} & n_{2} & \cdots & n_{N_{r}}\end{array}\right]^{T}$. The received signal at the receiver side is given by

$$
\begin{aligned}
\mathbf{y} & =\sqrt{\rho} \mathbf{H} \mathbf{x}_{j}+\mathbf{n}=\sqrt{\rho}\left(\sum_{k=1}^{N_{t}} \frac{1}{\sqrt{N_{t}}} \theta_{j, k} \mathbf{h}_{k}\right)+\mathbf{n} \\
& =\sqrt{\rho} \tilde{\mathbf{h}}_{j}+\mathbf{n},
\end{aligned}
$$

where $\rho$ denotes the average signal-to-noise (SNR) at each RA, $\mathbf{n}$ has independent and identically distributed (i.i.d.) entries according to $\mathcal{C N}(0,1)$, and $\tilde{\mathbf{h}}_{j}$ denotes the equivalent channel vector corresponding to the $j$-th VTA.

Unlike other SM schemes which convey information via physical TA indices [5]-[9], the proposed VSM conveys information by constructing VTA indices. For example as shown in Table I, $N_{t}=2$ physical TAs and $B=1$ bit APSs could construct $2^{B N_{t}}=4$ VTAs to realize 2 bits/symbol transmission, while conventional SSK could only transmit $\log _{2}\left(N_{t}\right)=1 \mathrm{bit} / \mathrm{symbol}$.
TABLE I

EXAMPLE OF THE VSM MAPPER RULE WITH $N_{t}=2$ AND $B=1$.

\begin{tabular}{c|c|c}
$\mathbf{b}=\left[\begin{array}{ll}b_{1} & b_{2}\end{array}\right]$ & VTA index $j$ & equivalent channel $\tilde{\mathbf{h}}_{j}$ \\
\hline$\left[\begin{array}{ll}0 & 0\end{array}\right]$ & 1 & $\tilde{\mathbf{h}}_{1}=\frac{1}{\sqrt{2}}\left(+\mathbf{h}_{1}+\mathbf{h}_{2}\right)$ \\
{$\left[\begin{array}{ll}0 & 1\end{array}\right]$} & 2 & $\tilde{\mathbf{h}}_{2}=\frac{1}{\sqrt{2}}\left(+\mathbf{h}_{1}-\mathbf{h}_{2}\right)$ \\
{$\left[\begin{array}{ll}1 & 0\end{array}\right]$} & 3 & $\tilde{\mathbf{h}}_{3}=\frac{1}{\sqrt{2}}\left(-\mathbf{h}_{1}+\mathbf{h}_{2}\right)$ \\
{$\left[\begin{array}{ll}1 & 1\end{array}\right]$} & 4 & $\tilde{\mathbf{h}}_{4}=\frac{1}{\sqrt{2}}\left(-\mathbf{h}_{1}-\mathbf{h}_{2}\right)$
\end{tabular}
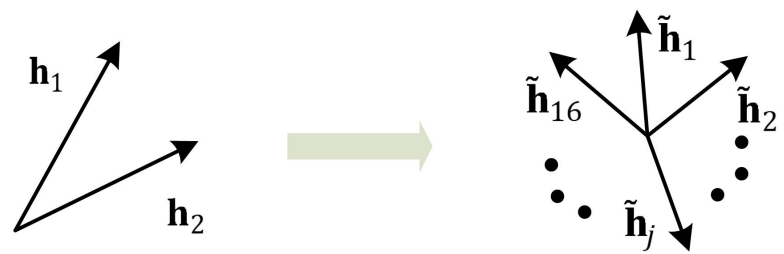

Fig. 2. Spatial constellation comparison of the convention SSK and the proposed VSM with $N_{t}=2$ and $B=2$.

\section{B. Spatial Constellation}

The spatial constellation of conventional SSK is actually the physical channel vectors corresponding to each physical TA [5], i.e.,

$$
\mathcal{X}_{\text {SSK }}=\left\{\mathbf{h}_{j}: 1 \leq j \leq N_{t}\right\},\left|\mathcal{X}_{\text {SSK }}\right|=N_{t} .
$$

Hence, the number of these spatial constellation points is directly determined by the number of physical TAs, and these spatial constellation points are uncontrollable. By adopting low-cost APSs with different phase rotation, the proposed VSM is able to provide a large number of spatial constellation points with a small number of physical TAs, i.e.,

$$
\mathcal{X}_{\mathrm{VSM}}=\left\{\tilde{\mathbf{h}}_{j}: 1 \leq j \leq 2^{B N_{t}}\right\},\left|\mathcal{X}_{\mathrm{VSM}}\right|=2^{B N_{t}} .
$$

By utilizing all available spatial constellation points, the modulation order (also transmit rate) could be significantly improved by the proposed VSM, i.e., $m_{\mathrm{VSM}} / m_{\mathrm{SSK}}=\frac{B N_{t}}{\log _{2}\left(N_{t}\right)}$.

For instance as shown in Fig. 2 with $N_{t}=2$ and $B=2$, the modulation order could be improved from 1 bits/symbol to 4 bits/symbol. Considering the same modulation order $m=1$, which means that there are only two spatial constellation points, we can carefully select them from all available candidates $\left(2^{B N_{t}}=16\right)$. Intuitively, the BER performance of the proposed VSM will be better than that of the conventional SSK due to the spatial constellation selection.

\section{Detection}

At receiver side, the detector assumes that the signal was transmitted by one out of $2^{B N_{t}}$ physical TAs, which are actually VTAs constructed by only $N_{t}$ physical TAs. Like in conventional SM [5], the detector's main function is to determine the virtual TA index. By assuming perfect CSI at receiver side, it is straightforward for the receiver to obtain the equivalent channel vectors as $\tilde{\mathbf{H}}=\left[\begin{array}{llll}\tilde{\mathbf{h}}_{1} & \tilde{\mathbf{h}}_{2} & \cdots & \tilde{\mathbf{h}}_{2 B N_{t}}\end{array}\right]^{T}$ based on Eq. (4).

By adopting these equivalent channel vectors, the optimal maximum likelihood (ML) detector [13] at the receiver can be 


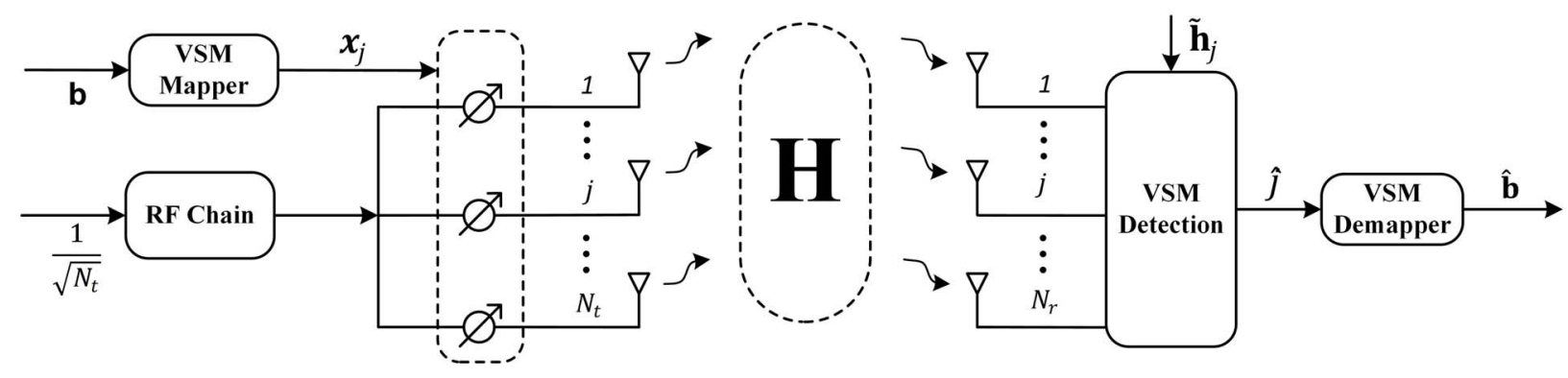

Fig. 1. The general system model of the proposed VSM.

represented as

$$
\begin{aligned}
\hat{j}_{\mathrm{ML}} & =\underset{j}{\arg \max } p_{\mathbf{Y}}\left(\mathbf{y} \mid \tilde{\mathbf{h}}_{j}\right)=\underset{j}{\arg \min }\left\|\mathbf{y}-\sqrt{\rho} \tilde{\mathbf{h}}_{j}\right\|_{\mathrm{F}}^{2} \\
& =\underset{j}{\arg \min } \sqrt{\rho}\left\|\tilde{\mathbf{h}}_{j}\right\|_{\mathrm{F}}^{2}-2 \operatorname{Re}\left\{\mathbf{y}^{H} \tilde{\mathbf{h}}_{j}\right\},
\end{aligned}
$$

where $\hat{j}_{\text {ML }}$ denotes the estimated active VTA index, and $p_{\mathbf{Y}}\left(\mathbf{y} \mid \tilde{\mathbf{h}}_{j}\right)$ is the probability density function (PDF) as

$$
p_{\mathbf{Y}}\left(\mathbf{y} \mid \tilde{\mathbf{h}}_{j}\right)=\frac{\exp \left(-\left\|\mathbf{y}-\sqrt{\rho} \tilde{\mathbf{h}}_{j}\right\|_{\mathrm{F}}^{2}\right)}{\pi^{N_{r}}} .
$$

In [13], a simple detector based on maximum ratio combining (MRC) is proposed, which could be directly applied to the proposed VSM as

$$
\hat{j}_{\mathrm{MRC}}=\underset{j}{\arg \max } \frac{\left|g_{j}\right|}{\left\|\tilde{\mathbf{h}}_{j}\right\|_{\mathrm{F}}^{2}},
$$

where $\mathbf{g}=\left[\begin{array}{llll}g_{1} & g_{2} & \cdots & g_{2^{B N_{t}}}\end{array}\right]^{T}=\tilde{\mathbf{H}}^{H} \mathbf{y}$ denotes the multiplying result of the Hermitian conjugate of the equivalent channel matrix and the received signal vector.

Moreover, sphere decoding (SD) based detector [14] and hybrid detector [15] (combining ML and MRC) could also be applied to the proposed VSM, which, however are omitted here for similarity. In summary, the detection complexity of the proposed VSM is actually the same as the conventional SSK with $2^{B N_{t}}$ physical TAs.

\section{Spatial Constellation Design}

In this section, the BER performance of the proposed VSM is firstly analyzed. Moving on, the selection of spatial constellation is formulated as a combinatorial optimization problem, and a SCO algorithm is provided to obtain a nearoptimal solution with low-complexity.

\section{A. BER Performance Analysis}

Given the specific channel matrix $\mathbf{H}$, the BER of the proposed VSM is union bounded [16] as

$$
\begin{aligned}
P_{e \mid \mathbf{H}} & =E_{i}\left\{\bigcup_{i, j} N(i, j) P\left(\tilde{\mathbf{h}}_{i} \rightarrow \tilde{\mathbf{h}}_{j} \mid \mathbf{H}\right)\right\} \\
& \leq \sum_{i=1}^{2^{B N_{t}}} \sum_{j=i+1}^{2^{B N_{t}}} \frac{2 N(i, j)}{2^{B N_{t}}} P\left(\tilde{\mathbf{h}}_{i} \rightarrow \tilde{\mathbf{h}}_{j} \mid \mathbf{H}\right),
\end{aligned}
$$

where $N(i, j)$ is the number of different bits between constellation points $\tilde{\mathbf{h}}_{i}$ and $\tilde{\mathbf{h}}_{j}, P\left(\tilde{\mathbf{h}}_{i} \rightarrow \tilde{\mathbf{h}}_{j}\right)$ denotes the pairwise error probability (PEP) of deciding on $\tilde{\mathbf{h}}_{j}$ given that $\tilde{\mathbf{h}}_{i}$ is transmitted, and the index in the summation is simplified since $N(i, j)$ is symmetric.

Based on the ML detector [13], the PEP could be obtained as

$$
\begin{aligned}
P\left(\tilde{\mathbf{h}}_{i} \rightarrow \tilde{\mathbf{h}}_{j} \mid \mathbf{H}\right) & =P\left(s_{j}>s_{i} \mid \mathbf{H}\right) \\
& =P\left(\frac{\operatorname{Re}\left\{\mathbf{n}^{H}\left(\tilde{\mathbf{h}}_{j}-\tilde{\mathbf{h}}_{i}\right)\right\}}{\left\|\tilde{\mathbf{h}}_{i}-\tilde{\mathbf{h}}_{j}\right\|_{\mathrm{F}}^{2}}>\frac{\sqrt{\rho}}{2} \mid \mathbf{H}\right) \\
& =Q\left(\sqrt{\kappa_{i, j}}\right),
\end{aligned}
$$

where $s_{i}=\frac{\sqrt{\rho}}{2}\left\|\tilde{\mathbf{h}}_{i}\right\|_{\mathrm{F}}^{2}-\operatorname{Re}\left\{\mathbf{y}^{H} \tilde{\mathbf{h}}_{i}\right\}$ denotes the ML decision criterion based on Eq. (7), and $Q(x)=\int_{x}^{\infty} \frac{1}{2 \pi} e^{-\frac{t^{2}}{2}} d t$. The parameter $\kappa_{i, j}$ can be represented as

$$
\kappa_{i, j} \triangleq \frac{\rho}{2}\left\|\tilde{\mathbf{h}}_{i}-\tilde{\mathbf{h}}_{j}\right\|_{\mathrm{F}}^{2} .
$$

Therefore, we can obtain the average BER of the proposed VSM as

$$
P_{e \mid \mathbf{H}} \leq \sum_{i=1}^{2^{B N_{t}}} \sum_{j=i+1}^{2^{B N_{t}}} \frac{2 N(i, j)}{2^{B N_{t}}} Q\left(\sqrt{\kappa_{i, j}}\right) .
$$

As indicated in [5], this union bound is quite loose due to the fact that the nearest neighbor constellations contribute more towards the PEP, which is averaged out by others in this analysis.

As an alternative to the union bound technique, another calculation method would be to only consider the nearest neighbor approximation of the PEP for a given channel matrix $\mathbf{H}$ [17], which can be expressed as

$$
P_{e \mid \mathbf{H}} \approx \lambda \cdot Q\left(\sqrt{\frac{\rho}{2} d_{\min }^{2}(\tilde{\mathbf{H}})}\right),
$$

where $\lambda$ is the number of neighboring constellation points, and $d_{\min }^{2}(\tilde{\mathbf{H}})$ is defined as

$$
d_{\min }^{2}(\tilde{\mathbf{H}})=\min _{i \neq j}\left\|\tilde{\mathbf{h}}_{i}-\tilde{\mathbf{h}}_{j}\right\|_{\mathrm{F}}^{2} .
$$

However, the neighboring number $\lambda$ could not be straightforwardly defined [5], [17], which is usually set as $\lambda=2$ in low modulation order. Although the BER bound is not represented as a close-form expression due to the fact that the PDF of 
neither $\kappa$, nor $d_{\min }^{2}$ could be easily obtained, we can find that for the ML detector, the BER performance is closely related to the EDs among selected spatial constellation points, especially the minimal one, i.e., $d_{\min }^{2}(\tilde{\mathbf{H}})$.

\section{B. Proposed Spatial Constellation Optimizing Algorithm}

According to Eqs. (12) and (15), we can find an intuitive suggestion from both bound expressions that the BER performance is closely related to the EDs among these equivalent channel vectors, especially the minimal one, i.e., $d_{\min }^{2}(\tilde{\mathbf{H}})$. This hint is actually quite similar with the constellation design principle in conventional APM [3], i.e., to maximize the minimal ED distance among constellation points.

Compared with the random selection of the spatial constellation (total $\left(\begin{array}{c}2^{B N_{t}} \\ 2^{m}\end{array}\right)$ kinds), the careful selection will certainly lead to BER gains by increasing the minimal ED among the selected spatial constellation points. Hence, given a modulation order $1 \leq m<B N_{t}$, the selection of the spatial constellation could be formulated as a combinatorial optimization problem $\mathcal{P}$ to maximize the minimal $\mathrm{ED}$, i.e.,

$$
\mathcal{P}: \mathcal{X}_{\mathrm{VSM}, \mathrm{opt}}^{(m)}=\underset{\mathcal{X} \in \mathcal{X}_{\mathrm{vSM},}, \mathcal{X X} \mid=2^{m}}{\arg \max } f(\mathcal{X}),
$$

where $f(\mathcal{X})=\min _{\tilde{h}_{i}, \tilde{h}_{j} \in \mathcal{X}, i \neq j}\left\|\tilde{\mathbf{h}}_{i}-\tilde{\mathbf{h}}_{j}\right\|_{\mathrm{F}}^{2}$ denotes the minimal ED among the spatial constellation $\mathcal{X}$. It is clear that such combinatorial optimization problem could be solved by exhaustive search among $\left(\begin{array}{c}2^{B N_{t}} \\ 2^{m}\end{array}\right)$ candidates with extremely high computational complexity.

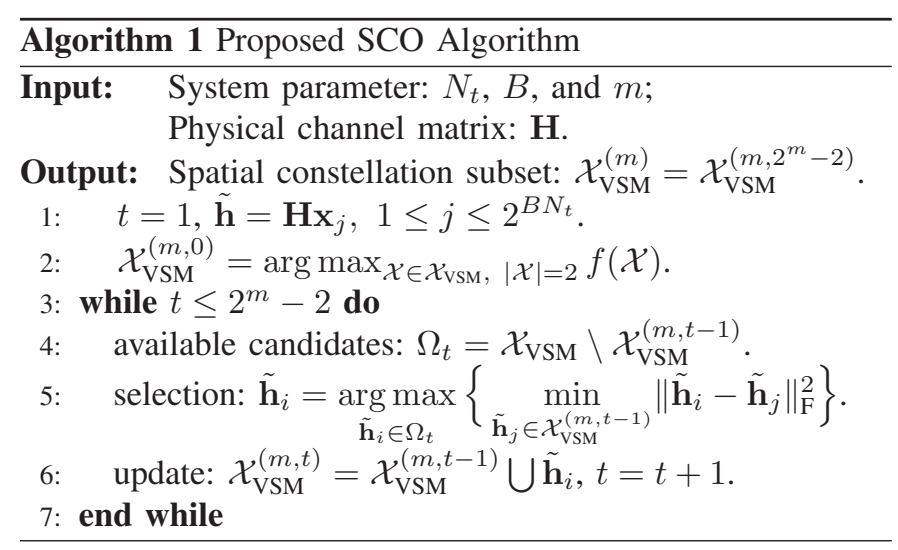

Applying common practice for adopting heuristic algorithm$\mathrm{s}$ to solve such combinatorial optimization problems, a SCO algorithm is proposed in this paper to provide a trade-off between the complexity and the performance. The key idea is to select the spatial constellation points in a sequential way to maximize the minimal ED among the currently selected spatial constellation points as

$$
\mathcal{P}_{t}: \mathcal{X}_{\mathrm{VSM}}^{(m, t)}=\underset{\tilde{\mathbf{h}}_{k} \notin \mathcal{X}_{\mathrm{VSM}}^{(m, t-1)}, \mathcal{X}=\mathcal{X}_{\mathrm{vSM}}^{(m, t-1)} \cup \tilde{\mathbf{h}}_{k}}{\arg \max } f(\mathcal{X}),
$$

where $\mathcal{X}_{\mathrm{vSM}}^{(m, t)}$ with $1 \leq t \leq 2^{m}-2$ denotes the selected spatial constellation point set at the $t$-th step, and $\mathcal{X}_{\mathrm{VSM}}^{(m, 0)}$ denotes

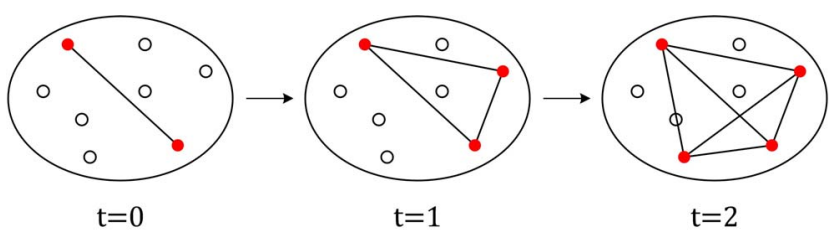

Fig. 3. An intuitive example for careful selection of the spatial constellation with $B=1, N_{t}=3$, and $m=2$.

the initial set having two spatial constellation points with the largest ED as

$$
\mathcal{P}_{0}: \mathcal{X}_{\mathrm{VSM}}^{(m, 0)}=\underset{\mathcal{X} \in \mathcal{X}_{\mathrm{vSM}},|\mathcal{X}|=2}{\arg \max } f(\mathcal{X}) .
$$

It is clear that the series of sequential subproblems $\mathcal{P}_{t}$ could be easily solved by traversing all candidates. This procedure will be carried out in a sequential way until all $2^{m}$ spatial constellation points are selected. The pseudocode of the proposed SCO algorithm is provided in Algorithm 1, whose physical meaning can be explained as follows.

1) Initialization (step 1-2): Based on the physical channel matrix $\mathbf{H} \in \mathbb{C}^{N_{r} \times N_{t}}$, the equivalent channel vectors $\tilde{\mathbf{h}}_{j}, 1 \leq$ $j \leq 2^{B N_{t}}$ are constructed, where each one corresponds to a VTA. Two spatial constellation points with the largest ED are firstly added into the initial set $\mathcal{X}_{\mathrm{vSM}}^{(m, 0)}$.

2) Sequential Selection (step 3-7): All $2^{m}$ spatial constellation points, but the first two will be selected in a sequential way. The spatial constellation points, which have not been selected, constitute the available candidate set $\Omega_{t}$ at step 4 . In step 5, we only calculate the minimal ED between the available candidates $\tilde{\mathbf{h}}_{i} \in \Omega_{t}$ and the current set $\mathcal{X}_{\mathrm{VSM}}^{(m, t-1)}$. Then, the candidate maximizing the minimal ED will be selected. At last, the selected spatial constellation point $\tilde{\mathbf{h}}_{t}$ will be added to form the current set $\mathcal{X}_{\mathrm{VSM}}^{(m, t)}$, and the iteration number $t$ increases. This procedure from step 3 to step 6 will be carried out for $2^{m}-2$ times until the final set is fulfilled as $\mathcal{X}_{\mathrm{VSM}}^{(m)}$.

In contrast to the exhaustive search which has to traverse all combinatorial candidates with computational complexity of $\mathcal{O}\left(\left(\begin{array}{c}2^{B N_{t}} \\ 2^{m}\end{array}\right) 2^{2 m}\right)$, the proposed SCO algorithm approximately discomposes the problem $\mathcal{P}$ into a series subproblems $\mathcal{P}_{t}$, which are much easier to be solved in a sequential way with significantly reduced computational complexity of $\mathcal{O}\left(2^{2 m+B N_{t}}\right)$. As shown in Fig. 3, an intuitive example for careful selection of the spatial constellation with $B=1$, $N_{t}=3$, and $m=2$ is illustrated. It is clear that only 3 steps are required to obtain the final spatial constellation with acceptable performance.

\section{Spatial Constellation Feedback}

Based on the pilot sequences from the transmitter, the receiver is able to acquire the CSI $\mathbf{H}$ (here we assume perfect CSI without noise for simplicity). Then, the equivalent channel vectors $\left\{\tilde{\mathbf{h}}_{j}: 1 \leq j \leq 2^{B N_{t}}\right\}$ could be constructed at the receiver. Considering modulation orders $1 \leq m<B N_{t}$, the spatial constellation set $\mathcal{X}_{\mathrm{VSM}}^{(m)}$ obtained at the receiver 


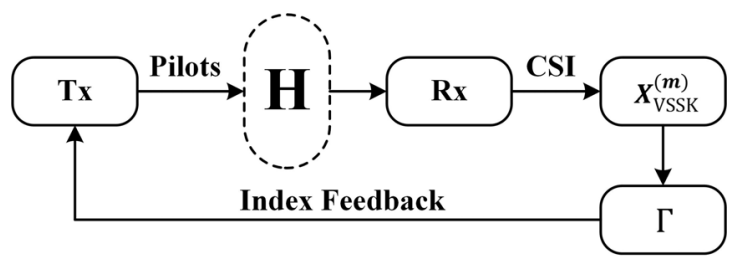

Fig. 4. The flow diagram of spatial constellation feedback in the proposed VSM scheme.

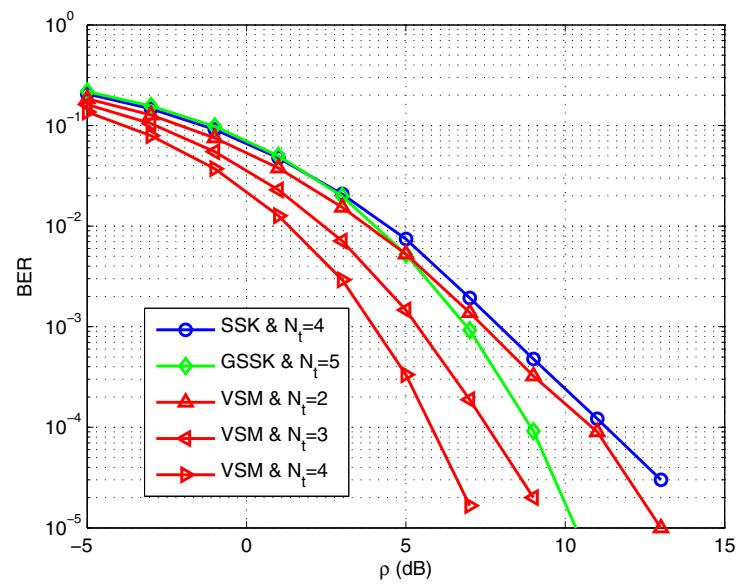

Fig. 5. BER performance of VSM versus SSK and GSSK for $m=2$ bps/symbol transmission with $B=1, N_{r}=4,2 \leq N_{t} \leq 5$, and ML detector.

by the proposed SCO algorithm has to be fed back to the transmitter. Fortunately, only the index set $\Gamma$ of the selected spatial constellation points in $\mathcal{X}_{\mathrm{VSM}}^{(m)}$ is required to be fed back instead of the whole equivalent channel vectors, i.e.,

$$
\Gamma=\left\{k: \tilde{\mathbf{h}}_{k} \in \mathcal{X}_{\mathrm{vSM}}^{(m)}\right\},|\Gamma|=2^{m} .
$$

This feedback procedure is as shown in Fig. 4, the overhead for only $2^{m}$ indices is quite small, which can be regarded as $2^{m}$ symbols of SE loss in practical systems [1], [2].

\section{Simulation Results}

In this section, several numerical cases are presented to compare the performance of VSM with state-of-the-art techniques. Monte Carlo simulation are performed for at least $10^{6}$ channel realizations. We consider a Rayleigh i.i.d. fading channel as described in Section II with perfect CSI at receiver. The plots illustrate the average BER performance versus $\rho$ (average SNR at per RA).

Fig. 5 demonstrates the BER performance of the proposed VSM versus the conventional SSK and GSSK for $m=2$ bps/symbol transmission with $B=1, N_{r}=4,2 \leq N_{t} \leq 5$, and ML detector. For the conventional SSK [5] and GSSK [7], $N_{t}=4,5$ are considered, while $2 \leq N_{t} \leq 4$ and $B=1$ are considered for the proposed VSM. It should be pointed out that the proposed SCO algorithm is adopted in VSM when $2^{B N_{t}}>2^{m}$ for spatial constellation design. By considering $N_{t}=4$ physical TAs, it is clear that a significant gain of 4

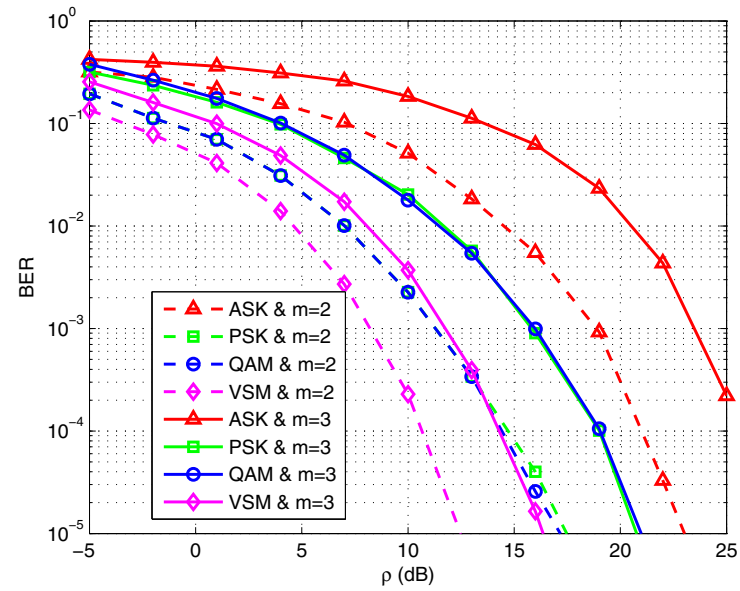

Fig. 6. BER performance of VSM versus ASK, PSK, and QAM with $B=1$, $N_{t}=N_{r}=4,2 \leq m \leq 3$, and ML detector.

$\mathrm{dB}$ at $P_{\mathrm{e}, \mathrm{bit}}=10^{-4}$ could be achieved over SSK. Even with only $N_{t}=2$ for the proposed VSM and $N_{t}=4$ for SSK, similar BER performance could be obtained, which indicates that the proposed VSM is able to reduce half of physical TAs and pilot overhead without BER performance degradation.

Fig. 6 shows the BER performance of the proposed VS$M$ versus amplitude shift keying (ASK), phase shift keying (PSK), and quadrature amplitude modulation (QAM) with $B=1, N_{t}=N_{r}=4,2 \leq m \leq 3$, and ML detector. For ASK, PSK, and QAM [3], $N_{t}=4$ the physical TAs transmit the same symbol as there is only one RF chain. It should be pointed out that random map is adopted for all considered modulation methods. These transmissions are under a total power constraint. It is clear that ASK performs worst, and a similar BER performance could be obtained by PSK and QAM. Compared with PSK and QAM with $m=2$ bits/symbol transmission, the proposed VSM is able to achieve a gain of $4 \mathrm{~dB}$ at $P_{\text {e,bit }}=10^{-4}$. Therefore, with only one RF chain, the proposed VSM is able to improve the BER performance compared with these APM schemes.

Fig. 7 illustrates the performance gain of the proposed SCO algorithm versus random selection with $B=1, N_{t}=N_{r}=$ 4 , and ML detector. Compared with the conventional SM schemes, the proposed VSM is able to provide a large number of spatial constellation points, i.e., $2^{B N_{t}} \gg N_{t}$. Hence, the proposed SCO algorithm is able to achieve a significant BER gain by selecting the spatial constellation points with large EDs in a greedy way. Considering $m=1 \mathrm{bps} / \mathrm{symbol}$ transmission, the BER performance gain of the proposed SCO algorithm is about $10 \mathrm{~dB}$ at $P_{\mathrm{e}, \mathrm{bit}}=10^{-4}$ compared with the random selection. By increasing the modulation order $m$, the BER performance gain is also reduced, and the same BER performance is obtained when $m=B N_{t}=4 \mathrm{bps} / \mathrm{symbol}$ transmission is considered, since all spatial constellation points are selected, i.e., two mauve diamond curves overlap. 


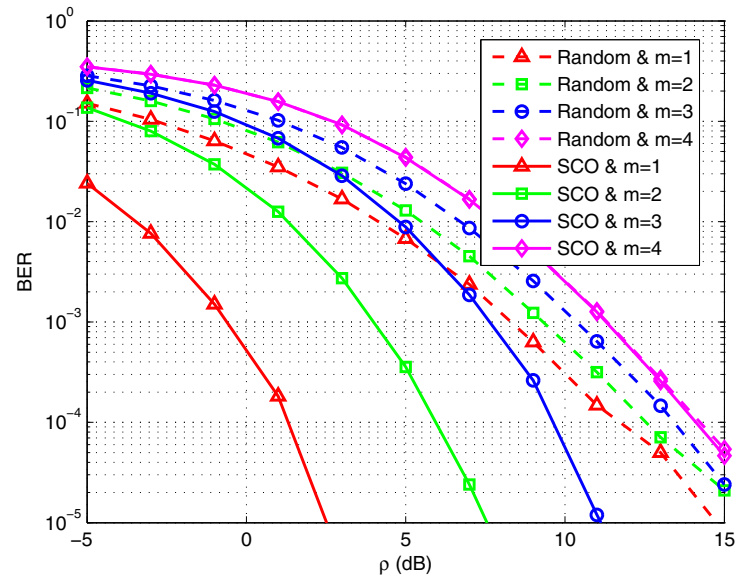

Fig. 7. Performance gain of the proposed SCO algorithm with $B=1$, $N_{t}=N_{r}=4,1 \leq m \leq 3$, and ML detector.

\section{CONCLUSIONS}

In this paper, we have developed a virtual spatial modulation (VSM) for MIMO systems, where the number of required TAs grow linearly with the number of transmitted bits. Our contribution is twofold: Firstly, we break away from the tradition SM schemes, which convey information by physical TA indices, and propose the concept of virtual TAs (VTAs). By applying low-cost APSs to construct exponentially growing VTAs, only linear physical TA increase (also linear pilot overhead increase) is required to achieve linear data rate increase. Secondly, we propose the concept of spatial constellation design to select spatial constellation points with large EDs from reconstructed equivalent channel vectors to improve the BER performance.

\section{ACKNOWLEDGMENT}

This work was supported in part by National High Technology Research and Development Program of China (Grant No. 2014AA01A704), in part by National Natural Science Foundation of China (Grant No. 61571267), in part by Beijing Natural Science Foundation (Grant No. 4142027), in part by Shenzhen Peacock Plan (No. 1108170036003286), in part by Shenzhen Visible Light Communication System Key Laboratory (ZDSYS20140512114229398), and in part by the UK Engineering and Physical Sciences Research Council (EPSRC) under Grant EP/K008757/1.

\section{REFERENCES}

[1] S. M. Alamouti, "A simple transmit diversity technique for wireless communications," IEEE J. Sel. Areas Commun., vol. 16, pp. 1451-1458, Oct. 1988.

[2] L. Hanzo, O. Alamri, M. El-Hajjar, and N. Wu, Near-capacity multifunctional MIMO systems: Sphere-packing, iterative detection, and cooperation. Hoboken, NJ, USA: Wiley, 2009.

[3] P. Fan and X. Xia, "A noncoherent coded modulation for 16QAM," IEEE Trans. Commun., vol. 49, no. 4, pp. 585-588, Apr. 2001.

[4] M. Di Renzo, H. Haas, A. Ghrayeb, S. Sugiura and L. Hanzo, "Spatial Modulation for Generalized MIMO: Challenges, Opportunities, and Implementation," in Proc. of the IEEE, vol. 102, no. 1, pp. 56-103, Jan. 2014.
[5] J. Jeganathan, A. Ghrayeb, L. Szczecinski, and A. Ceron, "Space shift keying modulation for MIMO channels," IEEE Trans. Wireless Commun., vol. 8, no. 7, pp. 3692-3703, Jul. 2009.

[6] R. Y. Mesleh, H. Haas, S. Sinanovic, A. W. Chang, and S. Yun, "Spatial modulation," IEEE Trans. Veh. Tech., vol. 57, no. 4, pp. 2228-2241, Jul. 2008.

[7] J. Fu, C. Hou, W. Xiang, L. Yan, and Y. Hou, "Generalised spatial modulation with multiple active transmit antennas," IEEE Glebecom Workshops, Miami, FL, USA, Dec. 2010, pp. 839-844.

[8] J. Wang, S. Jia, and J. Song, "Generalised spatial modulation system with multiple active transmit antennas and low complexity detection scheme," IEEE Trans. Wireless Commun., vol. 11, no. 4, pp. 1605-1615, Apr. 2012.

[9] J. Jeganathan, A. Ghrayeb, and L. Szczecinski, "Generalized space shift keying modulation for MIMO channels,", IEEE Int. Symp. Pers., Indoor, Mobile Radio Commun., Cannes, France, Sep. 2008, pp. 1-5.

[10] X. Wu, H. Claussen, M. Di Renzo and H. Haas, "Channel Estimation for Spatial Modulation," in IEEE Transactions on Communications, vol. 62, no. 12, pp. 4362-4372, Dec. 2014.

[11] A. Alkhateeb, G. Leus, and R. W. Heath, "Limited feedback hybrid precoding for multi-user millimeter wave systems," IEEE Trans. Wireless Commun., vol. 14, no. 11, pp. 6481-6494, Nov. 2015.

[12] O. El Ayach, S. Rajagopal, S. Abu-Surra, Z. Pi, and R. W. Heath, "Spatially sparse precoding in millimeter wave MIMO systems," IEEE Trans. Wireless Commun., vol. 13, no. 3, pp. 1499-1513, Mar. 2014

[13] J. Jeganathan, A. Ghrayeb, and L. Szczecinski, "Spatial modulation: Optimal detection and performance analysis," IEEE Commun. Lett. vol. 12, no. 8, pp. 545-547, Aug. 2008.

[14] A. Younis, R. Y. Mesleh, H. Haas, and P. Grant, "Reduced complexity sphere decoder for spatial modulation detection receivers," IEEE Global Commun. Conf., Miami, FL, USA, Dec. 2010, pp. 1-5.

[15] S. Sugiura, C. Xu, S. X. Ng, and L. Hanzo, "Reduced-complexity coherent versus non-coherent QAM-aided space-time shift keying," IEEE Trans. Commun., vol. 59, no. 11, pp. 3090-3101, Nov. 2011.

[16] J. G. Proakis, Digital Communications, (4th ed.) New York: McGrawHill, 2001.

[17] P. Yang, Y. Xiao, Y. Yi, and S. Li, "Adaptive spatial modulation for wireless MIMO transmission systems," IEEE Commun. Lett., vol. 15, no. 6, pp. 602-604, Jun. 2011 